\title{
Percepciones, Funciones y Significados del Consumo de Drogas en Jóvenes Escolares del Sector Oriente de Santiago
}

\section{Perception, Function and Meaning of Drug consumption in School youth of Eastern Zone of Santiago.}

\author{
Irene Magaña Frade * \\ Alicia Meschi Montaldo
}

\begin{abstract}
Resumen
Se presentan los resultados de un estudio de diseño mixto(con instrumentos cualitativos y cuantitativos), que es parte de un proyecto mayor de investigación /acción participativa, el cual se aplica en tres comunidades escolares privadas, correspondientes a un sector medio alto de la población del Área Oriente de Santiago.

La investigación estudió las percepciones, funciones y significados del consumo juvenil de drogas, discriminando factores de riesgo y protectores presentes en los colegios.

Los resultados abarcan diferentes aspectos y cruces de información. Se discriminan características, funciones y significados distintos al consumo de alcohol, marihuana y otras drogas. Respecto de los factores de riesgo, se diferencian algunos «generales» que se asocian a condiciones propias de cada colegio de otros más específicos relativos al contexto vincular inmediato de los jóvenes. Los factores protectores se relacionan a algunas condiciones internas de cada colegio que favorecen la salud mental juvenil. Finalmente, se concluye acerca de la importancia de éste tipo de estudios en la generación de campañas preventivas eficientes para la población escolar.
\end{abstract}

Palabras claves: Consumo juvenil de drogas, Investigación participativa, factores protectores, factores facilitadores.

\begin{abstract}
The study describes the different characteristics, functions, and significance related to drugs and alcohol consumption. The population participating in this research are three private school communities belonging to high-meddle social class, from Santiago de Chile.
\end{abstract}

* Académica de la Escuela de Psicología de la Universidad de Santiago de Chile. Chile, e-mail:imagana@lauca.usach.cl 
The results indicated the influence of both specific and shared community conditions on the populations' risk factor. In general terms, the risk factor is related to the general mental health of each community, and the principal conclusion is the necessity of using this kind of study in the design of prevention programs.

The authors utilized a mixed methodological design, combining qualitative and quantitative instruments within the research/action work frame.

Keys words: Juvenil drug consumption, participant research, protective factors, facilitating factors.

\section{Percepciones, Funciones y Significados del Consumo de Drogas en Jóvenes}

\section{Escolares del Sector Oriente de Santiago.}

El consumo juvenil de drogas es, actualmente, uno de los temas emergentes en el campo de la Salud Mental que promueve un mayor y más amplio debate social, político y científico académico. En torno a él se abren una serie de preguntas claves, que intentan cuestionarnos desde distintos modos de "entender" y "enfrentar" el polémico fenómeno; que hoy en día (dado su carácter conflictivo) se ha convertido en uno de los problemas sociales más críticos y urgentes de la contingencia nacional.

El consumo de drogas ha tenido presencia histórica en la mayor parte de las culturas y han ido variando las posiciones, criterios y políticas con que se han enfrentado sus significados, funciones y patrones de consumo. En Chile, la magnitud del problema está públicamente reconocida, ocupa titulares de prensa de manera cotidiana y a pesar de que sabemos tiene una masiva presencia en la población "joven" son notorias las carencias científicas y profesionales con que desde la Psicología respondemos al tema.

Las escasas publicaciones nacionales disponibles se centran fundamentalmente en estimaciones cuantitativas (Conace, 1998; Vicente y cols,1998; UNICEF, 1995.) y/o en algunos estudios descriptivos del consumo y sus factores asociados ( Florenzano 2000) que dan cuenta sólo de parte de los distin- tos aspectos de la ocurrencia del fenómeno. Otros estudios (Krause.M, Cornejo.M, Castillo.J,y cols 1999), nos informan también del profundo desconocimiento respecto de las motivaciones, funciones y distintas articulaciones subjetivas con que ocurre el consumo de drogas para los jóvenes; $y$, investigaciones internacionales (Choquet. $M$, Ledaux.S, 1994 Hoffman.L, Paris.S, Hall.E 1996) han documentado la gran diversidad de perspectivas teóricas y de aplicación con que desde las ciencias sociales se ha analizado el tema. La información disponible ha demostrado además la dificultad que estos abordajes parciales tienen para explicar en toda su magnitud el problema.

Obviamente, el fenómeno de las drogas tiene características, connotaciones y significados sociales diversos que oscilan desde una total estigmatización en el mundo adulto, hasta una banalización por parte de los jóvenes. Situación que tiende a invisibilizar su realidad y a confundir la posibilidad de acciones y políticas coherentes y efectivas para el tratamiento eficaz del problema.

En vista de la complejidad y polivalencia del tema y pensando que en la Salud Mental de un individuo convergen distintos factores psicosociales, familiares e individuales, el estudio del que aquí damos cuenta, se realiza como parte de una investigación mayor que desde un enfoque comunitario, se implementa en tres comunidades escolares del sector Oriente de Santiago; pensando que la escuela es espacio fundamental 
en donde transcurre la vida del joven y donde convergen también los adultos significativos en su desarrollo. En ella; jóvenes, padres y profesores conviven y se relacionan desde sus distintas realidades, posiciones e intereses y son los actores indispensables de cualquier modelo o plan de intervención para la prevención del consumo juvenil de drogas.

Debido a que el carácter operativo de una investigación en Salud Mental depende fundamentalmente del grado de inmediatez y de las posibilidades de aplicación de sus resultados, la investigación en que se enmarca este estudio, centra su interés en generar un modelo teórico práctico de investigación/acción que se aproxima a su objeto desde una perspectiva metodológica de tipo participativa, propia de la Psicología Comunitaria y que sirviéndose de un diseño mixto aúna, en sus procesos investigativos, tanto la posibilidad de comprender el problema cómo el promover cambios sobre él; generando un modelo de prevención e intervención eficiente para la comunidad escolar.

En esta comunicación solamente consideraremos los resultados globales de la fasediagnóstica del estudio, y nos proponemos tanto identificar los factores protectores y de riesgo asociados al consumo juvenil, como los significados y funciones que éstos cumplen en una comunidad escolar determinada.

\section{Método}

Metodológicamente, nuestro estudio, se enmarca en la investigación acción participativa (en la que se desarrolla toda la investigación), ya que teóricamente ésta supone servir tanto de instrumento de investigación como de medio de desarrollo y toma de conciencia del individuo. Sus procesos, en la medida que involucran a la gente en la identificación y resolución de sus problemas, elaboran experiencias personales y/o de grupo que, universalizándose para el ámbito donde se están desarrollando, tienen un impacto que sirve tanto a la construcción de modelos de acción como a la generación de cambios genuinos en la comunidad en la que ocurren.

De otra parte, la metodología participativa es capaz de integrar como un todo las vertientes cualitativas y cuantitativas del conocimiento y por tanto puede operar con herramientas e instrumentos que se integran a cualquiera de éstos órdenes de análisis. Los procedimientos de este método incluyen pasos y reglas propios de la investigación acción y considera válida la aplicación de diseños mixtos en los que se utilizan técnicas como una encuesta, grupos de discusión y entrevistas en profundidad. La recolección de los datos y el análisis de los mismos se desarrolla en forma paralela a la creación de estrategias de acción y no considera necesariamente momentos investigativos sucesivos.

\section{Participantes}

El proyecto fue realizado, paralelamente en tres comunidades escolares; caracterizadas por ser colegios particulares, pagados y mixtos del área oriente de Santiago. Todos ellos, agrupan a un sector de alumnos correspondientes al estrato medio alto, aunque presentan algunas diferencias: uno tradicional, británico, laico y considerado de alto nivel de exigencias académicas, integrado fundamentalmente por familias de empresarios y profesionales que se autodefinen orientados al éxito académico de sus hijos; otro católico, con énfasis en la formación valórica y la autodisciplina, cuyas familias corresponden en la mayoría a grupos progresistas en lo ideológico y político; y el tercero, abiertamente alternativo, con un esquema definido como de "educación en libertad", que tiende a agrupar familias con posturas ideológicas declara- 
damente de izquierda y, por tanto, muchas víctimas del daño producido por la represión y el exilio de la dictadura que terminó el año 1990.

La muestra del presente estudio estuvo compuesta por: 1) Todos los alumnos de $2 \square$ y $3 \square$ Medio de cada colegio - que suman un total de 651 sujetos- (que constituye la muestra a la que se le aplicó la encuesta). 2) Tres grupos de profesores constituidos por los profesores jefes de los cursos de $2^{\circ}$ y $3^{\circ}$ Medio y otros profesores de asignatura especialmente interesados en el tema - que suman un total de 40 sujetos-(que constituye la muestra del grupo de discusión de profesores) 3) Tres grupos de padres, reclutados en las reuniones de apoderados de $\operatorname{los} 2^{\circ}$ y $3^{\circ}$ medios, de acuerdo al interés y disponibilidad que ellos tenían- que suman una cantidad de 45 sujetos-(constituyen la muestra del grupo de discusión de padres) y 4) Tres grupos de alumnos que se integraron en base a "representantes" de los distintos grupos que integraban cada curso de $2^{\circ}$ y $3^{\circ}$ medio de cada uno de los colegios - que suman una cantidad de 48 sujetos-(constituyen la muestra del grupo de discusión de alumnos). Además, constituyeron la muestra 9 sujetos mas, todos considerados sujetos claves, "conocedores" de la realidad del consumo al interior del colegio, quienes fueron entrevistados y participaron como "expertos" en una discusión sobre los resultados.

\section{Instrumentos y Recolección de Datos.}

La recolección de datos se realizó usando distintos instrumentos de investigación (cuantitativos y cualitativos), que permitieron rescatar un máximo de información acerca de la realidad de cada comunidad escolar estudiada. A éste efecto se aplicó una encuesta y se realizaron varias sesiones de trabajo con distintos grupos de discusión (de padres, profesores y alumnos) y además, se hicieron una serie de entrevistas en profundidad.

La encuesta fue previamente construida por las investigadoras, considerando los supuestos e intereses específicos del estudio. Consta de 12 preguntas estructuradas y semiestructuradas y una escala de tipo Likert (30 proposiciones); todas relativas a la percepción, conocimiento y significados que los estudiantes tienen $\mathrm{y}$ adscriben al consumo juvenil de drogas. La encuesta refiere sus objetivos a: 1) Conocer los distintos tipos de drogas legales e ilegales que consumen los jóvenes y discriminar la magnitud del consumo de cada una, diferenciando porcentualmente por frecuencia entre consumidores "excesivos", "moderados" y "ocasionales". 2) Determinar características del contexto de consumo, así como las motivaciones que lo sustentan. 3) Conocer la relación entre consumo juvenil de drogas y la percepción del joven de variables familiares tales como; acuerdo, permisividad, expectativas y exigencias parentales; grado de satisfacción del joven con la relación existente con el padre, madre y hermanos; actitud de los padres frente al consumo de drogas y adecuación del joven a las reglas de convivencia en el hogar. 4) Conocer la relación entre consumo juvenil de drogas y la percepción del joven de variables sociales y relacionales con sus pares. 5) Conocer la relación entre el consumo juvenil de drogas y la percepción del joven de variables individuales tales como: autoimagen, estilo de resolución de problemas, y creencias acerca de los significados y funciones del alcohol y otras drogas. 6) Conocer la relación entre el consumo juvenil de drogas y la percepción del joven de variables escolares tales como rendimiento escolar y relación con los profesores..

Los grupos de discusión investigaron las percepciones, creencias, temores, actitudes, etc. de los padres, profesores y alumnos 
respecto del consumo juvenil de drogas; ya que como técnica permiten recabar no sólo las opiniones presentes en un determinado grupo, sino también detectar las estructuras de sentido que se revelan a partir de la interacción entre sus miembros. Con la técnica empleada se esperaba que en las interacciones los sujetos sometieran sus primeras opiniones a reflexión y crearan así un discurso compartido que permitiera una mejor comprensión de su propia incertidumbre cuando hablaban sobre el consumo juvenil de drogas. Se usó una pauta de temas eje y se generaron varias sesiones de trabajo, tendientes a profundizar y consensuar la opinión de los grupos. La investigación produjo y analizó 9 grupos de discusión, obedeciendo a la finalidad propia de la metodología cualitativa de llegar a una sistematización de la información que logre cubrir la diversidad empírica de los discursos.

Las entrevistas investigaron las percepciones y opiniones de algunos sujetos cuya posición clave les daba acceso privilegiado a información sobre la dinámica, el contexto y las características del consumo en cada una de las comunidades escolares estudiadas. Las entrevistas se realizaron en forma flexible, formulando preguntas que fueron modificadas en función de las respuestas de cada uno de los sujetos. Se pretendió explicar y comprender el fenómeno del consumo a partir de los significados y las definiciones personales que transmitían los entrevistados. Se realizaron un total de 9 entrevistas.

\section{Procedimiento}

La investigación se realizó en tres fases. Una primera "fase funcional" que permite básicamente consolidar los grupos de trabajo y socializarlos en la metodología participativa, comprometiéndolos en lo que significan las acciones preventivas. Una se- gunda fase "diagnóstica-autodiagnóstica", cuyos resultados son de lo que damos cuenta en este artículo y que en su recolección de datos se extendió a 4 meses. En esta fase se aplica la encuesta y se centra el trabajo de los grupos en la identificación de la problemática relativa al consumo específico de cada comunidad particular. En esta fase también se realiza la identificación de personas "claves" y las entrevistas en profundidad. Una tercera fase "operativa" que, integrando los diferentes actores (alumnos, padres y profesores) genera un nuevo grupo de trabajo que se centra en elaborar estrategias de acción claves para la posterior implementación de un programa preventivo.

Después de la aplicación de la encuesta, se trabajó con los grupos de discusión el tema relativo a las relaciones inter e intra estamentos, los sentimientos que éstas dinámicas generan, y su posible influencia en el consumo juvenil de drogas. Asimismo, se revelaron en forma diferenciada los problemas, necesidades e intereses de los jóvenes, padres y profesores para entender desde ahí cómo éstos se articulan en al problemática del consumo de drogas. Posteriormente, y en cada uno de los grupos de trabajo, se realizó un análisis de los resultados de la encuesta en términos de los tipos y niveles de consumo, y de los factores de riesgo que en ella se perfilaron.

\section{Análisis de datos}

El análisis de datos se realizó: para la encuesta según el Programa estadístico SPSS, versión 10.0 para Windows. Para los grupos de discusión se usa un análisis de discurso que considera el reconocimiento de unidades de sentido mediante el criterio de saturación (considerando la determinación de cada unidad cuando los discursos pronunciados permiten cerrar con relativa coherencia una estructura de 
sentido, y cuando los nuevos discursos no alteran la unidad de esa estructura). Para las entrevistas se realiza un primer análisis de tipo descriptivo, extrayendo formulaciones que funcionan como hipótesis que permiten articular analíticamente el resto del material obtenido por técnicas cualitativas, y que son contrastadas en momentos posteriores del análisis con el resto de los datos.

\section{Resultados}

A continuación se presentará una síntesis de los resultados mas relevantes a cada una de las áreas temáticas abordadas por el presente estudio, los que en conjunto dan cuenta de las percepciones y significados que los estudiantes, padres y profesores tienen sobre los factores involucrados en el consumo juvenil de drogas ( en las comunidades escolares de nuestro estudio). Además, con un cruzamiento de los datos, se realiza un análisis por cortes transversales de algunos ejes temáticos: a) dinámicas internas de la comunidad escolar, b) problemas, necesidades y percepciones de alum- nos, padres y profesores, c) características y significados del consumo de drogas en los colegios, y d) sistematización de la información y conclusiones.

En este análisis transversal se consideran tanto los datos obtenidos en las distintas comunidades escolares como aquellos obtenidos a través de los diferentes instrumentos.

\section{Del Consumo de Drogas en las Comuni- dades Estudiadas}

Respecto a los niveles de consumo de drogas, en la encuesta se utilizaron las siguientes categorías de frecuencia: Consumo excesivo (más de dos veces a la semana); consumo moderado (una a dos veces a la semana); consumo ocasional (menos de dos veces al mes); no consumo (nunca).

Los tres colegios estudiados se comportaron de manera diferente respecto al consumo juvenil de drogas.

\begin{tabular}{ll}
\hline Colegio menor consumo Total & \multicolumn{1}{c}{ Colegio mayor consumo total } \\
\hline $\begin{array}{l}\text { Mayor consumo moderado de Alcohol } \\
\text { Mayor consumo ocasional de alcohol }\end{array}$ & $\cdot$ Mayor consumo excesivo de alcohol \\
\hline$\cdot$ Mayor consumo ocasional de Marihuana & $\begin{array}{l}\cdot \text { Mayor consumo excesivo de Marihuana } \\
\cdot \text { Mayor consumo moderado de Marihuana }\end{array}$ \\
\hline $\begin{array}{l}\text { Menor consumo ocasional de otras sustan- } \\
\text { cias }\end{array}$ & $\begin{array}{l}\cdot \text { Mayor consumo ocasional de otras sustan- } \\
\text { cias }\end{array}$ \\
\hline
\end{tabular}

Fig. 1 Perfil de los colegios de menor y mayor consumo total.

Lo que determino un perfil respecto al comportamiento del consumo de cada droga como el siguiente 


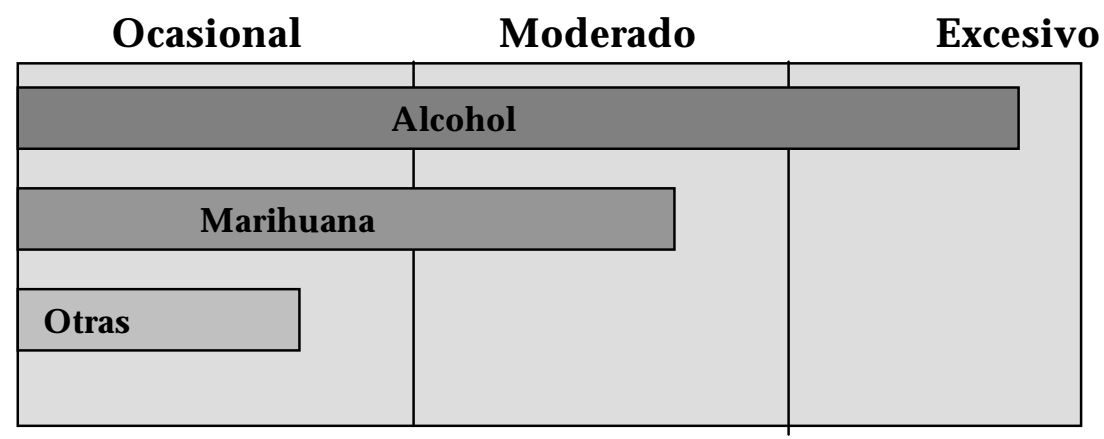

Fig. 2 Comportamiento del Mayor consumo

El consumo de alcohol varió en un rango muy estrecho en las tres comunidades, correspondiendo el menor consumo excesivo $(7 \%)$ al colegio de menor consumo total $(77 \%)$, y también el mayor consumo excesivo $(15,7 \%)$ al colegio de mayor consumo total $(83,1 \%)$.

El consumo de marihuana, por el contrario, se diferenció claramente en los tres colegios: $16,2 \%, 34,5 \%$ y $47,7 \%$. Cabe destacar que en el colegio de mayor consumo total, el $60,8 \%$ de los consumidores se ubicaron en los rangos de mayor consumo excesivo y moderado. En los otros dos, se invirtió la situación, logrando el consumo ocasional casi duplicar a las categorías ex- cesiva y moderada. El comportamiento de la marihuana, entonces, es muy diferente del alcohol. En primer lugar, las características del consumo no parecen obedecer a un patrón sociocultural común sino a factores internos de la comunidad escolar. En segundo lugar, cuando la marihuana adquiere presencia social en el grupo de jóvenes, pareciera producirse una pérdida del control de la forma que asume el consumo - control representado por el consumo ocasional - y una mayor aceptación, tolerancia y apertura a la droga. Este fenómeno se retroalimenta a su vez en una secuencia circular con el aumento de la frecuencia de consumo.

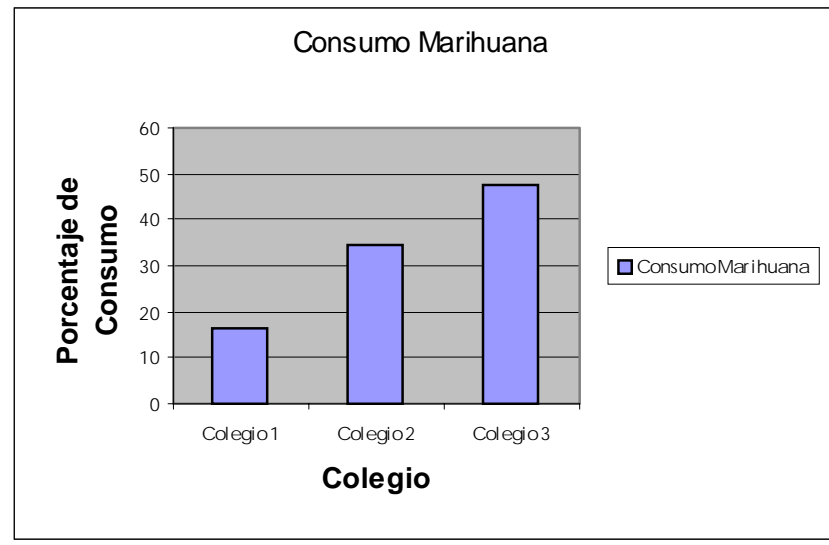

Fig. 3 Perfil del consumo de Mh por colegio. 
El consumo de anfetaminas y tranquilizantes fue considerablemente mas bajo en todos los colegios - entre un $1.7 \%$ y $7.5 \%$ , siendo este consumo casi exclusivamente ocasional. El consumo de cocaína, también de carácter ocasional, fluctuó entre un $1.3 \%$ y $4.7 \%$. Podríamos concluir que en los colegios estudiados el consumo de otras drogas ha ingresado sólo marginalmente y no constituye aún un gran problema.

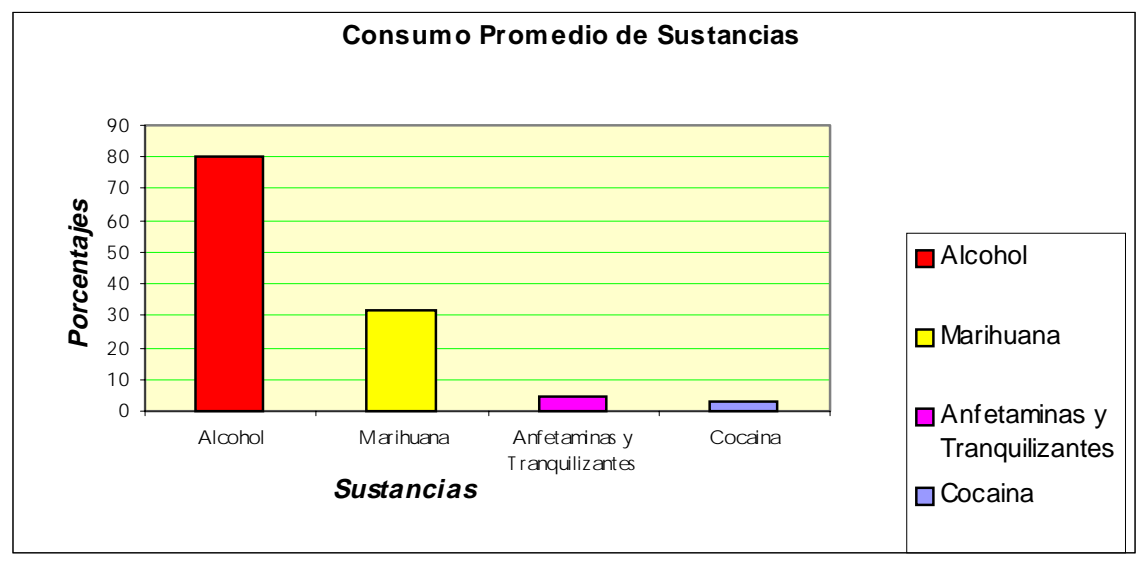

Fig. 4 Perfil por promedio del consumo de drogas.

Finalmente, se encontró una alta correlación entre el consumo de alcohol, marihuana y otras drogas, lo cual confirman los datos existentes en la literatura de que a mayor consumo de alcohol, mayor consumo de otras drogas ilegales.

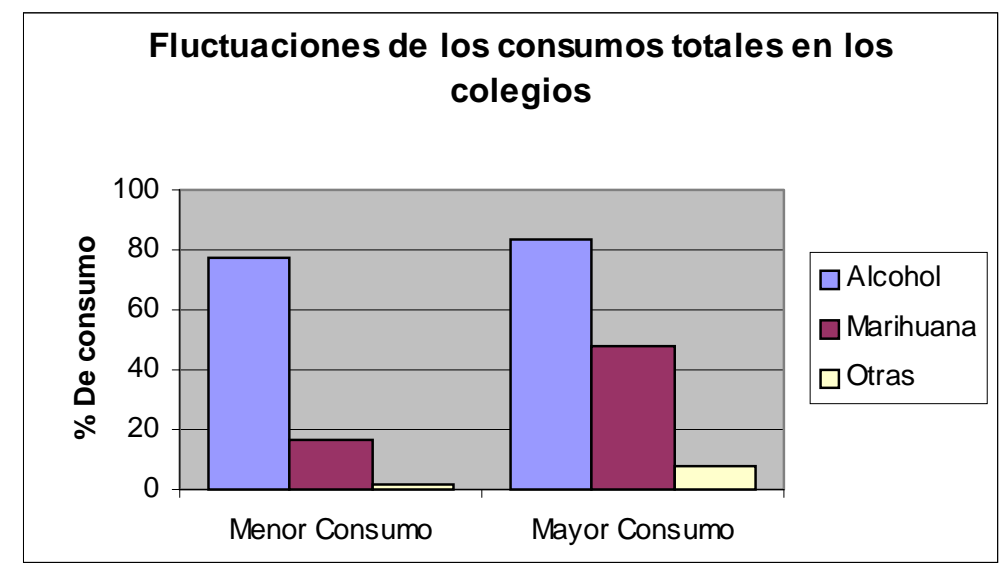

Fig. 5 Perfil de menor y mayor consumo total por tipo de drogas.

\section{Del contexto del consumo de marihuana}

El consumo de marihuana se da preferentemente en "calle, parque, mirador". Los jóvenes se apropian de los espacios abier- tos que, es posible, que simbólicamente representen la falta de estructuras y normas externas, o la libertad deseada por el "mundo juvenil". Como segunda alternativa, mencionan las "fiestas" (en aquellos cole- 
gios donde el consumo es mas frecuente) y las "casas de amigos" (en aquel colegio donde el consumo es menor y más oculto). Pareciera que a mayor connotación negativa adscrita a la droga, mayor clandestinidad y anonimato en su consumo. Por el contrario, a mayor consumo, mayor aceptación y menor temor y cuidados por consumir en situaciones mas abiertas y públicas.

Aunque la mayoría de los jóvenes consume menos de un "pito" en cada ocasión, existe una tendencia a aumentar la cantidad a medida que aumenta la frecuencia del consumo. Esto confirma el supuesto teórico de la alta correlación existente entre frecuencia y cantidad de consumo, y demuestra que el consumo es "excesivo" en ambos sentidos.

Mas del 75\% de los jóvenes que consumen marihuana lo hacen "con amigos"; son sobre todo los consumidores "excesivos" los que consumen "con la pareja" o "solo". Lo cual significa, en nuestra opinión, que en este último tipo de consumo se pierde el carácter y las motivaciones sociales relacionales propias del consumo más ocasional, predominando variables personales y necesidades subjetivas y afectivas íntimas.

La mayoría de los jóvenes dice consumir marihuana porque "tenía ganas", algunas veces porque quería "pasarlo bien", pero nunca porque el "grupo decidió consumir", "no me atreví a decir que no" o "me sentía bajoneado". Da la impresión, entonces, que la motivación exteriorizada del consumo de marihuana estaría mas relacionada a las necesidades evolutivas de autoafirmación, búsqueda de vivencias nuevas y placente- ras, etc.; $y$, aparentemente, menos relacionada con las carencias y problemas emocionales y sociales.

Cerca del 50\% del consumo de marihuana se acompaña con alcohol, lo cual reafirma el estrecho vínculo existente entre ambas drogas. El hecho de que sean fundamentalmente los consumidores ocasionales los que consumen marihuana con alcohol, y sobre todo los consumidores excesivos los que consumen sólo marihuana, nos hablaría de una evolución en la forma y dinámica del consumo; desde el consumo más social y abierto, al mas personal y privado. Esta situación nos permite hipotetizar en el sentido de la existencia de motivaciones de tipo hedonistas, evasivas o incluso simplemente adictivas.

Finalmente, entre un $66 \%$ y un $84 \%$ de los padres desconoce el consumo de marihuana de sus hijos. A menor consumo de marihuana en la comunidad escolar, menor conocimiento de los padres del consumo de los hijos. Y de los padres que saben el consumo de sus hijos, la mitad son padres de consumidores excesivos. Estos resultados nos confirman el carácter clandestino del consumo de marihuana y la desconexión entre el mundo adulto y el mundo juvenil. Además, parece que a medida que aumenta la frecuencia del consumo, éste tiende a hacerse más evidente, perdiéndose los controles y cuidados anteriores. Pareciera que la ilegalidad y el tabú de la marihuana, junto con la falta de contacto entre adultos y jóvenes, deja a éstos últimos en una situación de abandono y riesgo que sólo ellos pueden intentar revertir, una vez que el consumo ha alcanzado niveles alarmantes. 


\section{De los padres, profesores y jóvenes, y de las relaciones entre ellos.}

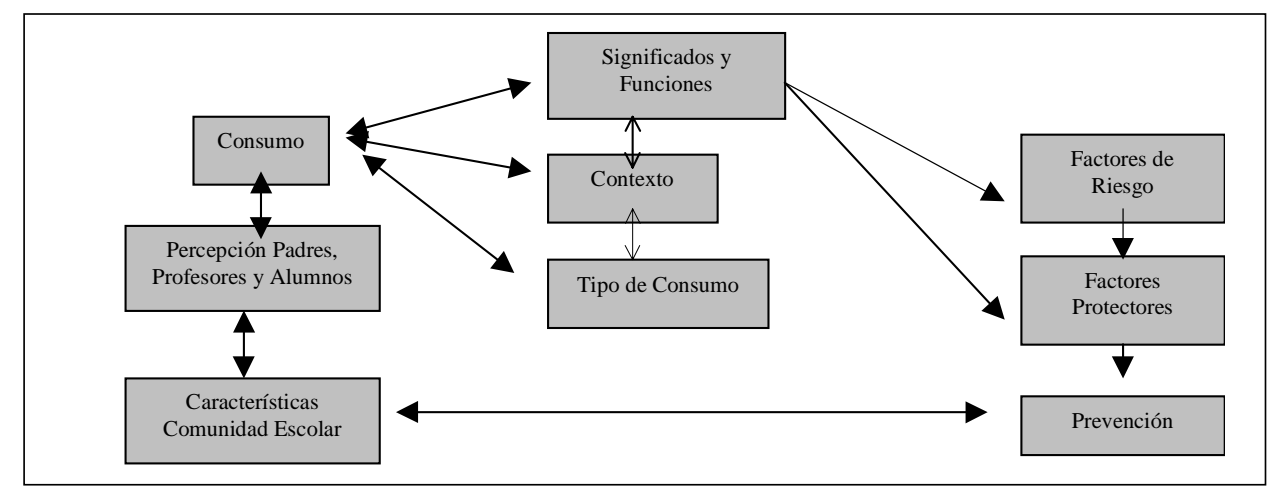

Fig. 6 Aspectos involucrados en la percepción y significados del consumo para padres, profesores y jóvenes.

En los distintos grupos de discusión, los padres participantes aparecieron muy sensibles al problema del consumo juvenil de drogas y muy preocupados de la situación de riesgo en que perciben a sus hijos. La droga les produce una sensación de amenaza difusa y les provoca reacciones que oscilan desde el miedo a que, el hijo, sea involucrado y se convierta en adicto hasta la autoconvicción tranquilizadora de que, éste, será capaz de resistir el influjo de la droga. Esta inquietante gama de respuestas va casi siempre acompañada de un "no saber qué hacer" para evitar que la amenaza se cumpla.

Inmersos en esta conflictiva insegurizante, los padres manifiestan la necesidad de buscar apoyo para tratar de "controlar" la situación, esperando entonces del estudio herramientas concretas e inmediatas que, en el contexto social y escolar de sus hijos, les permita tener una mayor sensación de control y aliviar así su ansiedad.Lo cual a su vez, trae como consecuencia la toma de conciencia de la necesidad y urgencia de un trabajo organizado conjunto en torno a la prevención de drogas.

Los profesores, de otra parte, expresan también confusión e impotencia ante la sensación de exigencia de cumplir con tareas de tipo formativas en lo valórico, dada la percepción de sobrecarga de trabajo y de horario a la que se sienten sometidos. En este contexto, la perspectiva de participar en un programa preventivo, aunque sentida como necesaria e interesante desde el punto de vista profesional, viene a aumentar la sensación de sobrecarga y cansancio crónico.

Sin embargo, los profesores en general se muestran ansiosos de recibir información y preparación que les permita detectar e intervenir en casos de jóvenes que se encuentran en situación de "verdadero riesgo"; es decir, que estén actualmente consumiendo drogas ilegales. Se le asigna entonces gran importancia a este tipo de intervención secundaria, en desmedro de una prevención primaria en favor de la promoción de la Salud Mental.

La demanda de obtener apoyo especializado para cumplir funciones que en realidad no les corresponde como profesores ya que no son ni los padres ni los terapeutas de los jóvenes- puede ser fácilmente acogida si no se está atento y conciente del riesgo que implica esta superposición de roles, que potencia la fantasía del abandono parental y aleja e incapacita más aún a los padres. Sin embargo, el apoyo requerido puede orien- 
tarse a potenciar sus recursos como educadores respecto a los jóvenes y a coordinar su acción con los padres y otros adultos significativos, así como con otras instituciones de la comunidad más amplia, respetando los límites y los campos de influencia.

Los jóvenes, finalmente, participaron en el programa desde una postura predominantemente marcada por el escepticismo y un bajo nivel de responsabilidad y compromiso general. Esta postura pareciera fundamentarse, entre otros, en la sensación que ellos tienen de que su opinión y experiencia generalmente no es válida para los adultos. Aunque usualmente son llamados a participar en distintos ámbitos de actividades, éstas finalmente resultan definidas por los adultos, trasluciendo su concepción de los jóvenes y limitándolos entonces a insertarse en un marco de referencia que les resulta ajeno. Esta realidad permanente de exclusión está determinando la actitud de "no estar ni ahí" y la vivencia de un "mundo juvenil" construido desde la automarginación, la desconfianza, el desencantamiento, y la falta de credibilidad en el "mundo adulto".

Por otro lado, y en el contexto socioeconómico y cultural de la población escolar participante en la experiencia, nos encontramos con jóvenes "acomodados" y acostumbrados a satisfacer " fácil y rápidamente" sus necesidades y deseos, a la vez que educados en un sistema claramente vertical y directivo donde la regla esperada consiste en cumplir con las expectativas de la autoridad adulta.

Sin embargo, presionados por una metodología que exige una autorreflexión acerca de sus necesidades, y una búsqueda activa de los posibles caminos para satisfacerlas en el contexto de su realidad social más cercana, los jóvenes se mostraron deseosos de hacer conciente y exteriorizar sus carencias y conflictos más importantes. En cada colegio, el autodiagnóstico así elaborado por los jóvenes sorprendió a su comunidad de padres y profesores, los cuales no sólo desconocían aspectos esenciales de sus dificultades sentidas, sino que los idealizaban, imaginándolos más satisfechos y seguros en un ambiente familiar y escolar "protegido " y "positivo". Esta "ceguera" responde probablemente a la necesidad de los adultos de "hacerlo bien" y de que sus esfuerzos fructifiquen en "niños felices". Por lo tanto, ellos se sienten casi "traicionados " al enfrentar la realidad expresada por los jóvenes, llegando en algunos casos a cuestionar la veracidad de los contenidos más dolorosos.

Fueron también los jóvenes los más duros y mordaces en su crítica hacia sí mismos, los adultos y su sistema escolar, develando siempre ellos los "secretos", lo que está subyacente y que no se habla, lo "oculto". Permitiendo así el conocimiento y comprensión de las dinámicas más profundas que los afectan en el sistema de relaciones que los rodea e incluye.

Las reacciones frente a la información entregada por las investigadoras sobre los resultados de la encuesta se diferenciaron en los grupos de discusión y por colegios. Todos las Grupos de Alumnos (de los tres colegios), consideraron que el consumo real, especialmente de marihuana y otras drogas ilegales, era mayor que el aparecido en la encuesta. Ellos esgrimieron como argumento explicativo la falta de confianza y poca credibilidad en la palabra de los adultos y, por tanto, en el carácter anónimo de la encuesta.

Los Grupos de Profesores, en base a su experiencia, también creían que el consumo sobrepasaba las cifras de la encuesta; sin embargo, mientras que en dos colegios, ellos aceptaron aliviados estos resultados, en el tercero insistieron enfáticamente que el consumo era mayor y desconfiaron, abiertamente, de las respuestas de los jóvenes.

Los Grupos de Padres fueron los únicos que prácticamente no tenían información 
respecto al consumo juvenil de drogas en sus colegios ( el tema era tabú), excepto en uno de ellos donde el consumo era mas abierto. Por lo tanto, las reacciones frente a los resultados de la encuesta tuvieron una mayor connotación emocional, ya que estaban autorreferidas a las fantasías y deseos proyectados en el comportamiento de los hijos, y variaron según las características familiares de cada población escolar, pasando desde la angustia hasta la negación.

\section{De los Factores de Riesgo}

El trabajo de investigación en los tres colegios nos permitió relevar algunas condiciones facilitadoras del consumo.

La primera condición facilitadora es una baja coherencia interna de la organización "comunidad escolar", que se manifiesta fundamentalmente en la falta de transparencia, inconsistencias entre el discurso y la acción, falta de claridad y/o contradicciones dentro y entre los distintos estamentos, e inconsistencia en la aplicación de normas. Esto parece crear un clima de confusión, inseguridad, falta de credibilidad y desconfianza que constituye un factor de alta vulnerabilidad para los jóvenes.

Por otra parte, las expectativas adultas no explícitas, ambiguas o paradojales, así como la falta de exigencias y presiones para cumplir con expectativas ya definidas, también contribuyen a la creación de un contexto desestructurante que dificulta la formación de identidad y la generación de proyectos claros en los jóvenes. La otra cara de la medalla, es una organización, cuyo "deber ser" rígido y estereotipado define inflexiblemente los roles y comportamientos deseados y adecuados en los jóvenes, lo que también les dificulta el ser diferentes, los presiona a la igualdad y a la adecuación a las normas y criterios grupales, empujándolos necesariamente a la sobreadaptación al grupo.
Así las presiones para cumplir con los mandatos sociales y/o familiares excesivamente rígidos y sobrevalorados, puede dificultar la diferenciación de los jóvenes y el logro de una identidad personal. Aquellos del tipo "sean felices", "sean libres", dado su carácter paradojal, deja a los jóvenes intrínsecamente vulnerables al efecto de la droga, la cual los hace sentirse transitoriamente "libres" y "felices", permitiéndoles así cumplir fácilmente con el mandato, quedando sin embargo tan atrapados como antes. Por otro lado, aquellos del tipo "sean exitosos", en el caso que los jóvenes no tengan todas las condiciones personales y ambientales para lograrlo, producen sentimientos de frustración, impotencia y desvalorización que pueden ser aliviados por el efecto evasivo de la droga.

La ausencia de proyectos claros y viables en los jóvenes que, independientemente de donde surjan, pudieran hacerles sentido, motivarlos y darles coherencia a su quehacer cotidiano, constituye otro factor de riesgo. Es decir, sin metas que canalicen y sustenten sus energías, quedan inmersos en la "lata" y a merced de cualquier atracción transitoria que llene sus espacios y tiempos vacíos.

También los déficit en el cumplimiento adulto de las funciones de protección y contención, unidos a intentos de control inadecuados, deja a los jóvenes apoyados en "muros de goma", sin cimientos estructurales que le den el respaldo y seguridad necesarios en su proceso de individuación y autonomía. La droga, entonces, produce un efecto que les proporciona la sensación de pseudoautonomía y pseudoindividualización.

La falta de estructuras en la comunidad escolar que canalicen las demandas y necesidades de los jóvenes y les permitan encontrar un espacio de protección y ayuda en situaciones personales problemáticas, los deja una vez más referidos solamente a sus pares como fuente de ayuda. 
Otro aspecto es la experiencia de pérdidas individuales y sociales que no han podido ser elaboradas ni resueltas adecuadamente en el contexto sociopolítico actual, genera un clima emocional básicamente depresivo, definido por sentimientos de desmotivación, indiferencia, descontento, rabia e impotencia. En este ambiente colectivo, los jóvenes tienden a la tristeza, soledad y la sensación de que "todo vale callampa", abrumados por problemas que los trascienden.

Asimismo, en un clima emocional distante y frío - caracterizado por dificultades en la expresión de emociones y afecto, poca comunicación y confianza - la droga cumple la importante función de "entibiar" las relaciones humanas y aumentar la cercanía, creando así una sensación de pseudointimidad.

Generalmente, asociado a este clima de distancia afectiva, se da también la separación rígida y estereotipada entre lo femenino y lo masculino, con predominio de represiones e inhibiciones en las relaciones entre jóvenes del sexo opuesto. Junto con "entibiar" el ambiente, la droga desinhibe a los jóvenes, les facilita el contacto heterosexual y les posibilita comportamientos con el sexo opuesto que no se podrían permitir sin ella.

Finalmente, todos los factores de riesgo anteriormente descritos tienen alguna relación con el desarrollo de la autonomía, la cual es condición necesaria y movilizadora del proceso de formación de identidad juvenil. Entendemos entonces que el consumo de drogas cumple la función fundamental de hacer sentir al joven que está realizando sus procesos de diferenciación e individualización propios de la etapa adolescente, cuando en realidad está inmerso en una situación ilusoria que lo mantiene atrapado y dependiente, incapaz de reconocer y enfrentar sus dificultades reales para el logro de sus tareas evolutivas.

\section{De los factores especificos que aumen- tan la frecuencia del consumo de dro- gas en los jóvenes.}

Al hablar de factores específicos, pensamos en situaciones tanto internas como del contexto vincular inmediato más significativo de los jóvenes, que influyen en el aumento y mantención de un alto nivel de consumo de drogas. Estos factores surgieron del análisis de los resultados de la encuesta, al relacionar los datos específicos de los grupos de consumo "excesivo" de alcohol y "moderado" y "excesivo" de marihuana con diferentes variables individuales, familiares y escolares; y cómo éstas, eran percibidas por los jóvenes.

A nivel familiar, los factores que aparecen asociados a estos niveles de consumo de alcohol y marihuana son: Poca claridad en las reglas de convivencia familiar; falta de respeto por las normas; estilo permisivo de control parental; algún grado de aceptación y tolerancia del consumo de drogas por parte de los padres; baja concordancia entre las expectativas parentales y el comportamiento real del joven; familia uniparental; mayor consumo de drogas del hermano mayor; poca armonía percibida en la relación conyugal entre los padres; baja satisfacción en la relación con los padres; relación con el padre caracterizada por mayor conflicto y menor cercanía, aceptación, comunicación, comprensión y confianza; relación con la madre caracterizada por menor valoración, comprensión y confianza.

En el ámbito escolar, se detecta una menor satisfacción con el rendimiento y una sensación de menor aceptación, comprensión, valoración y apoyo de parte de los profesores.

En los grupos de pares, aparece una relación entre el consumo propio y el consumo de los amigos. Por otra parte, se observa una tendencia a sentir mayor satisfac- 
ción en la relación con los amigos y el sexo opuesto - y una menor satisfacción con los padres y profesores.

Respecto de las creencias, los grupos más consumidores perciben el consumo de alcohol como algo "normal", y las drogas "ilegales" (marihuana, anfetaminas y cocaína) como menos dañinas y peligrosas.

Esta constelación de factores de riesgo nos permiten hipotetizar que nuestros jóvenes consumidores se mueven en un determinado contexto psicosocial que los mantiene en una situación de vulnerabilidad frente a la droga: Problemas de límites entre los subsistemas parental y filial; débiles y/o inadecuadas pautas de control e inconsistencia en los patrones de disciplina; carencias en la comunicación y contacto entre padres e hijos; modelos adultos poco confiables y eficientes, clima de mayor aprobación y tolerancia al consumo de drogas como forma de enfrentar problemas y revertir estados emocionales "negativos"; distintos tipos y grados de conflictos intrafamiliares. Asimismo, las dificultades en la relación con padres y profesores y los problemas de rendimiento escolar generan en estos jóvenes sentimientos de ser menos queridos, aceptados, respetados, valorados, comprendidos y dignos de confianza por parte de "mundo adulto", afectando así su autoestima y aumentando su dependencia del grupo de pares como única fuente de pertenencia y continente que brinda valoración y apoyo incondicional.

\section{De los Factores Protectores.}

Respecto de factores protectores, cada comunidad escolar demostró poseer condiciones internas favorables a la Salud Mental de su población, las que fue posible relevar como recursos internos que protegían a los jóvenes del riesgo de consumir drogas.

En relación con la estructura y dinámica de la institución escolar, destacan caracte- rísticas tales como: coherencia interna, flexibilidad, participación, eficiencia, recursos técnico-pedagógicos, excelencia académica.

La amplitud y variedad de actividades extracurriculares, de espacios de convivencia y desarrollo social de los jóvenes posibilitan la integración grupal, la identificación y sentido de pertenencia con el colegio.

Otros factores se refieren al cuerpo directivo y docente: funcionamiento como equipo de trabajo; buen nivel técnico y profesional; motivación y compromiso con la formación valórica de los alumnos; cercanía y calidez en la relación profesor- alumno; estilo docente democrático y participativo.

Respecto a los apoderados, resultan favorables: una organización de padres comprometida con el colegio y dispuesta a aportar en la formación valórica de los alumnos; una población de padres suficientemente protectores, capaces de cumplir con sus funciones parentales.

Finalmente, respecto a los alumnos, aparecen como factores protectores: una población juvenil con motivaciones de logro; con posibilidades de realizar esfuerzos y rendir de acuerdo a sus intereses; capaz de juicio crítico y autocrítico; capacidad de contactarse emocionalmente con los propios problemas y posibilidad de expresar el descontento.

\section{Conclusiones}

Si buscáramos el espacio ideal para hacer una prevención eficiente del consumo juvenil de drogas con un enfoque comunitario de promoción de la Salud Mental, que privilegie a los jóvenes como actores y receptores fundamentales de estas acciones preventivas, nos encontraríamos en los patios y salas de un colegio. Es allí donde es posible aunar e integrar las distintas posturas así como los esfuerzos de todos los ac- 
tores involucrados en el esfuerzo preventivo - jóvenes, profesores, directores, padres, orientadores y psicólogos, personal administrativo y de servicio.

Esta experiencia, ha comprobado nuestro supuesto inicial respecto al carácter único y específico de cada comunidad escolar, el cual determina y sustenta un tipo de consumo de drogas con niveles y características propios y diferentes. Al sumarse éstos con otros factores macrosociales a los procesos de búsqueda de identidad y crecimiento propios de la etapa juvenil, se crea una situación especial de vulnerabilidad interna para los jóvenes de cada comunidad que en definitiva favorece y explica su consumo de drogas.

A la luz de estos resultados, surge inevitablemente la necesidad de que todo programa de prevención del consumo juvenil de drogas, incluya una primera etapa diagnóstica participativa que integre los conocimientos, percepciones, sentimientos y aportes de los sujetos involucrados. Son estos elementos cualitativos y cuantitativos los que permiten armar un cuadro que refleje ampliamente las características relevantes de la comunidad y las condiciones de vulnerabilidad con que los jóvenes se insertan en ella En la investigación pudimos diferenciar dos tipos de factores de riesgo: aquellos generales, que nos permiten comprender el sustento en que se enmarca el consumo juvenil de drogas; y que está íntimamente vinculado a las dinámicas relacionales internas del colegio, y los factores más específicos o de "alto consumo" que se refieren a dinámicas familiares e individuales. Los primeros son más característicos de cada comunidad escolar, mientras que los segundos son más específicos a los grupos de alto consumo y, por lo tanto, están presentes en el universo de consumidores "excesivos" de todos lo colegios.

Desde el punto de vista de la prevención, este resultado implica que el diseño de los programas debe diferenciarse en cada colegio de acuerdo a su realidad respecto de los "factores comunitarios" y los "factores protectores", y debe contener también líneas de acción comunes que respondan a los "factores de alto consumo".

Respecto a los tipos de drogas y los niveles de consumo encontrados podemos decir que en los colegios estudiados, no existe un consumo juvenil preocupante de drogas ilegales más "duras" que la marihuana. Pareciera que la variable "miedo" está actuando como una protección que mantiene a los jóvenes alejados de este tipo de consumo, a pesar de que el tráfico está presente en su medio ambiente.

El alcohol, en cambio, aparece como omnipresente, sobrevalorado, incluso fomentado, y funcional a una inserción social y grupal adaptativa y cohesionante. La presión cultural para consumir bebidas alcohólicas es precoz y se extiende a todos los contextos, especialmente hacia el género masculino. Dada su connotación trivial y/o positiva en el mundo adulto, pierde totalmente el carácter de "droga", y se introduce abierta y festivamente en casi todos los ámbitos recreativos juveniles. Su consumo, entonces, es masivo y no discrimina entre tipos de jóvenes; casi todos en algún momento consumen.

Por el contrario, el consumo de la marihuana es más o menos oculto - dependiendo del contexto comunitario -; es decir, su consumo está más restringido al grupo de pares o de amigos, y ocurre preferentemente lejos de la presencia y el control de los adultos. Su función está asociada con motivaciones de diferenciación y transgresión, expresando más rebeldía y "choreza" que adecuación a las normas y expectativas sociales adultas. Respecto a la escalada del consumo de drogas, este estudio nos indica que es el alcohol el que abre la puerta a las otras drogas ilegales. Los consumidores de alcohol se inician en la marihuana como consumidores "ocasionales" y, en la medida que incrementan este consumo ile- 
gal y se convierten en consumidores "excesivos", tienden a hacerlo en forma exclusiva, sin acompañarlo de alcohol.

A diferencia del alcohol, el consumo de marihuana parece responder primordialmente a "factores comunitarios", lo cual explicaría las grandes diferencias en los niveles y características del consumo en los tres colegios. Aunque los "factores de alto consumo" de todas maneras perfilan en forma comprensible a los consumidores "moderados" y "excesivos" de marihuana, igual como sucede con los consumidores "excesivos" de alcohol.

Las distintas características del consumo de alcohol y marihuana nos hacen concluir que es necesario diferenciar claramente las estrategias preventivas para las drogas legales e ilegales.

Basándonos en los resultados de esta investigación, proponemos que la prevención del consumo de alcohol pase, prioritariamente, por un trabajo con los adultos más cercanos como modelos consumidores, para que su acción en los jóvenes tenga éxito; mientras que la prevención de la marihuana y otras drogas ilegales sea dirigida más directamente a los grupos de jóvenes, aunque sin dejar de involucrar la responsabilidad que les cabe a los adultos.

Creemos que este estudio es sólo el comienzo del hilo conductor que puede llevarnos a desentrañar la complejidad del problema "consumo juvenil de drogas" y sus significados más profundos. Se hace necesario continuar investigando y re-inventar nuevos caminos para entender mejor los "factores de alto consumo", diferenciar las formas en que se instalan en el comportamiento juvenil las distintas drogas y los distintos tipos de consumo, así como conocer alguno de los mecanismos de influencia de las variables socio-económicas, culturales, familiares, del grupo de pares, de los modelos adultos, de los medios de comunicación, etc.

Finalmente, resulta fundamental concebir los programas preventivos que se realicen a nivel escolar desde una perspectiva comunitaria que permita revelar los "factores comunitarios" que sustentan la funcionalidad del consumo de drogas en cada uno de los colegios.

\section{Referencias Bibliográficas}

Choquet, M.; Ledoux, S.(1994). Analyses et Prospective Adolescents.Enquete national. París: Les Editions Inserm.

CONACE. Estudio Nacional del consumo de alcohol, tabaco y drogas en la población escolar de Chile (de $8^{\circ}$ a $4^{\circ}$ medio). Informe final. Chile, 1998.

FLORENZANO (2000) http:// www.unne.edu.arl / cyt / $2000 / 1$ _sociales/spolf.s_031.pdf

Hoffman, L.; Paris, S.; Hall, E.(1996). Psicología del desarrollo hoy. España: McGrawHill.

Krause,M.; Cornejo, M.; Castillo, J.; Soto, A.; Calderón, R.; Guerra, P.;Guerrero, P.; Y MACKenZIE, M. (1999): Representaciones Sociales del consumo de drogas en jóvenes. Revista Psykhe, Vol.8. $\mathrm{N}^{\circ} 1$, Mayo, 93 -99.

UNICEF, Fundación Paz Ciudadana, Minsal, Mineduc (1995). Consumo de Alcohol, Tabaco y Drogas en Adolescentes de la Región Metropolitana.

Vicente, P.; Rioseco, P.; Vielma, M.; Saldivia, S.; Madariaga, C. (1998); Abuso y dependencia de sustancias psicoactivas en Chile (1998). Rev. De Psiquiatría; (2): 7080 .

Período de límite de recepción de artículos: 30 de Marzo 2002. Fecha de aceptación artículos: 30 de octubre 2002. 